\title{
How Price-Based Frequency Regulation Impacts Stability in Power Grids: A Complex Network Perspective
}

\author{
Peng Ji $\mathbb{D}^{1,2,3,4}$ Lipeng Zhu $\mathbb{D}^{5},{ }^{5}$ Chao Lu, ${ }^{6}$ Wei Lin $\mathbb{D}^{1,2,3}$ and Jürgen Kurths ${ }^{4,7,8}$ \\ ${ }^{1}$ Institute of Science and Technology for Brain-Inspired Intelligence, Fudan University, Shanghai 200433, China \\ ${ }^{2}$ LCNBI and LMNS (Fudan University), Ministry of Education, Shanghai 200433, China \\ ${ }^{3}$ Research Institute of Intelligent and Complex Systems, Fudan University, Shanghai 200433, China \\ ${ }^{4}$ Potsdam Institute for Climate Impact Research (PIK), 14473 Potsdam, Germany \\ ${ }^{5}$ Department of Electrical and Electric Engineering, The University of Hong Kong, 999077 Hong Kong, China \\ ${ }^{6}$ Department of Electrical Engineering, Tsinghua University, 100084 Beijing, China \\ ${ }^{7}$ Department of Physics, Humboldt University, 12489 Berlin, Germany \\ ${ }^{8}$ Saratov State University, 83, Astrakhanskaya Str., 410012 Saratov, Russia \\ Correspondence should be addressed to Peng Ji; pengji@pik-potsdam.de and Lipeng Zhu; zhulpwhu@126.com
}

Received 9 October 2019; Revised 23 January 2020; Accepted 31 January 2020; Published 28 February 2020

Academic Editor: Chittaranjan Hens

Copyright $\odot 2020$ Peng Ji et al. This is an open access article distributed under the Creative Commons Attribution License, which permits unrestricted use, distribution, and reproduction in any medium, provided the original work is properly cited.

\begin{abstract}
With the deregulation of modern power grids, electricity markets are playing a more and more important role in power grid operation and control. However, it is still questionable how the real-time electricity price-based operation affects power grid stability. From a complex network perspective, here we investigate the dynamical interactions between price-based frequency regulations and physical networks, which results in an interesting finding that a local minimum of network stability occurs when the response strength of generators/consumers to the varying price increases. A case study of the real world-based China Southern Power Grid demonstrates the finding and exhibits a feasible approach to network stability enhancement in smart grids. This also provides guidance for potential upgrade and expansion of the current power grids in a cleaner and safer way.
\end{abstract}

\section{Introduction}

Climate change mitigation requires us to transform current energy systems, a major shift over the next few decades in how energy is produced and transferred in a cleaner way [1]. In modern deregulated power grids, electricity markets provide substantial benefits for energy conservation and emission reduction, especially with the help of various means of advanced metering and communications $[2,3]$. Particularly, with the real-time based electricity price in electricity markets, competitive activities and thus abundant interactive phenomena are introduced into physical power networks [4]. Due to such complex interactions and low efficiency of analysis in large-scale grids, however, power system engineers and operators are still prone to getting trouble in a systematic analysis of interactive phenomena, especially in system stability analysis [5], although the power engineering community has made tremendous efforts to address these problems for years.

In the fields of complex systems and complex networks, considerable attempts have also been devoted to an efficient analysis of rich interactive and collective phenomena $[6,7]$ in power grids. Various models of power systems have increasingly been brought to higher attention recently, partially thanks to the better understanding of collective phenomena such as synchronization of power grids [7-11]. In particular, the Kuramoto model as a paradigm for general oscillator models has been intensively investigated recently [7], from the perspective of networked control [10], selforganized synchronization [12-17], to stability against even large perturbations $[18,19]$. The further investigation of the Kuramoto model with or without inertia attracts great interest from the aspects of, e.g., the optimal placement of virtual inertia for system performance [20] and for perfect 
synchronization $[21,22]$, non-Gaussian frequency fluctuations described by Levy-stable laws [23]. From the viewpoint of power grid operators, it is vital yet challenging to enhance network stability in complex power grids, especially in the presence of electricity markets [4]. In particular, how the interplay between networked dynamics and real-time price regulations affects power grid stability still remains unanswered.

Here, to cope with these problems, we adopt the typical governing dynamics of power systems, the second-order Kuramoto model but additionally including generation/consumption adaption to electricity price regulation. This is intended to maximize energy conservation and respective economic benefits and to quantify impacts of price-based dynamics on the network against even large perturbations. Our experimental results first in a low-dimensional system indeed suggest that the frequency adaption to price regulation can enhance system's stability, but the adaption can also reduce its stability. Next, we show that these findings remain valid in the real world-based China Southern Power Grid (CSG) where the existing thermal, hydro, and nuclear generation and various typical consumers are included. Furthermore, we find that, after duly decreasing the percentage of thermal generation and accordingly increasing hydrogeneration's percentage by improving economic efficiency to meet the total load demands, not only the environmental contamination is mitigated but the network also remains fairly stable.

\section{Results}

2.1. Governing Dynamics. In the context of power grids, electricity markets play a crucial role in demand side response and economic dispatch of generation. Given an appropriate governing mechanism of the real-time electricity price in the electricity market, both generators and loads are inspired to adjust their generation and consumption activities accordingly, resulting in an energy conservation and an emission reduction, as well as possible enhancement of system stability. Taking into account both physical network dynamics and market effects, all the dynamics of price regulations, node responses, and networked interactions are expressed as follows.

The time-varying price is mainly designed for frequency regulation. Supposing the price at a given time $t$ is $p(t)$, its control law follows [24]:

$$
p(t)=p_{N}-K_{P}\left[e(t)+\frac{1}{T_{l}} \int_{0}^{t} e(\tau) \mathrm{d} \tau\right],
$$

where $p_{N}$ denotes the rated price in a steady state (without frequency deviation); $K_{P}$ and $T_{l}$ are the main parameters of the control law; and $e(t)$ represents the average frequency deviation of the whole network, serving as the feedback signal of the overall network dynamics. In particular, $e(t)$ is estimated as [25]

$$
e(t)=L_{f}\left(\sum P_{g i}-\sum P_{d j}\right),
$$

where $L_{f}$ is the linear coefficient relating the amount of active power imbalance of the whole network to the average frequency deviation and $P_{g i}$ and $P_{d j}$ (with implicit subscript $t$ ) stand for the generated and consumed active power by the $i$ th generator and the $j$ th load, respectively. $L_{f}$ is calculated as $L_{f}=f_{N} /\left(\mathrm{MS}_{N}\right)$, where $f_{N}$ is the rated frequency $(50 \mathrm{~Hz}$ in China and Europe and $60 \mathrm{~Hz}$ in the USA), $M$ is the average inertia of all the generators, and $S_{N}=\sum P_{g i}$ is the total rated generation capacity of the whole network in the steady state.

For a certain power supplier, denoted as $i$, given the current output $P_{g i}$, the rate of expansion and/or reduction of its production is adjusted in proportion to the difference between the real-time price $p(t)$ and its marginal cost $p_{g i}$ [26]. Such a response behavior yields

$$
\tau_{g i} \frac{\mathrm{d} P_{g i}}{\mathrm{~d} t}=-p_{g i}+p(t)
$$

where $\tau_{g i}$ represents the time constant of the response of the supplier $i$. Qualitatively, $\tau_{g i}$ is determined by an array of elements, including technical features, the working status for the time instant of receiving the price signal, and the means of communication of these signals [26]. Especially, technical features, primarily involving unit ramp rates, seem to have a main effect on the response time.

Intuitively, marginal cost $p_{g i}$ corresponds to the increment of cost with one-unit increase of the output $P_{g i}$. Given the cost function of a generator, denoted by $C\left(P_{g i}\right), p_{g i}$ is estimated by the derivative of $C\left(P_{g i}\right)$. In terms of thermal generation, supposing its output is $P_{g t}$, the corresponding cost $C\left(P_{g t}\right)$ can be simply approximated as a quadraticfunction form $[27,28]$ :

$$
C\left(P_{g t}\right)=\alpha_{t}+\beta_{t} P_{g t}+\gamma_{t} P_{g t}^{2},
$$

where $\alpha_{t}, \beta_{t}$, and $\gamma_{t}$ are the coefficients of the function. Then, the marginal cost $p_{g}\left(P_{g t}\right)$ is computed by

$$
p_{g}\left(P_{g t}\right)=\beta_{t}+2 \gamma_{t} P_{g t}=b_{g t}+c_{g t} P_{g t},
$$

where $b_{g t}$ and $c_{g t}$ denote the constant and monomial coefficients of the linear marginal-cost function, respectively. Therefore, considering the generation cost with a quadratic-function form [27, 28] (see supplementary note (SN) 1), the marginal cost is determined by $p_{g i}=b_{g i}+c_{g i} P_{g i}$.

The consumer $j$ responds to the time-varying price $p(t)$ in a similar manner as above, reducing its consumption once $p(t)$ exceeds its marginal benefit under the current consumption $P_{d j}$. Specifically, it is described as

$$
\tau_{d j} \frac{\mathrm{d} P_{d j}}{\mathrm{~d} t}=p_{d j}-p(t)
$$

where $\tau_{d j}$ and $p_{d j}$ correspond to its time constant of response and marginal benefit. Given a quadratic function of the consumption benefit $[27,28]$, analogous to the marginal cost $p_{g j}$, the consumer's marginal cost is computed by $p_{d j}=b_{d j}+c_{d j} P_{d j}$.

We use the classical power grid model $[9,12,19,29,30]$, in coarse scale, where each oscillator $i$ is characterized by generation $P_{i}=P_{g i}$ or consumption $P_{i}=-P_{d i}$, and its governing dynamics follows: 


$$
\begin{aligned}
& \frac{\mathrm{d} \theta_{i}}{\mathrm{~d} t}=\omega_{i}, \\
& \frac{\mathrm{d} \omega_{i}}{\mathrm{~d} t}=-\alpha \omega_{i}+P_{i}-\sum_{j=1}^{N} K_{i j} \sin \left(\theta_{i}-\theta_{j}\right),
\end{aligned}
$$

where $\theta_{i}$ and $\omega_{i}$ are the instantaneous phase and frequency of the $i$ th oscillator, $\alpha$ is the viscous damping coefficient, and the line capacity $K_{i j}=K_{j i}$ accounts for topological connections, with $K_{i j}>0$ if nodes $i$ and $j$ are connected and $K_{i j}=0$, otherwise.

Interactions between different kinds of dynamics are illustrated in the schematic diagram (Figure 1(a)). Particularly, generations $P_{g i}(3)$ and consumptions $P_{d i}(6)$ are intercoupled with price regulation (1). Equations (1)-(6) can affect the node dynamics (7), but not vice versa. Rich phenomena arise due to the competition between the generated power $P_{i}$ and the synchronization forcing term summing neighbors' power delivery $K_{i j} \sin \left(\theta_{i}-\theta_{j}\right)$ along the transmission lines $[12,19,30]$. Functional reasons require that the limit of the transmission capacity $K_{i j} \gg P_{i}$, which is though uneconomical. However, with the rapid growth of power demands these years, $P_{i}$ is expected to be extremely near but less than $K_{i j}$ to avoid costly transmission system expansion. Grids therein are operated at high risk of instability and even inducing blackout when suffering from large disturbances.

2.2. Two-Node Model. As an illustration to reveal the underlying phenomena and mechanisms, we first consider a simple two-node model consisting of one generator (denoted by subscript $g$ ) and one consumer (denoted by subscript $d$ ). Their phase difference $\Delta \theta=\theta_{g}-\theta_{d}$ and frequency difference $\Delta \omega=\omega_{g}-\omega_{d}$ satisfy from (7):

$$
\begin{aligned}
& \frac{\mathrm{d} \Delta \theta}{\mathrm{d} t}=\Delta \omega, \\
& \frac{\mathrm{d} \Delta \omega}{\mathrm{d} t}=-\alpha \Delta \omega+\Delta P-2 K \sin (\Delta \theta),
\end{aligned}
$$

where $\Delta P=P_{g}+P_{d}$ and $K_{g d}=K_{d g}=K$, with constant price $\lambda=\lambda_{N}=\left(b_{d} c_{g}-b_{g} c_{d}\right) /\left(c_{g}-c_{d}\right)$ and power generation balanced by consumption $P_{g}=P_{d}=P_{N} \equiv\left(b_{d}-b_{g}\right) /\left(c_{g}-\right.$ $c_{d}$ ) in the stationary state.

The governing dynamics of $\Delta P$ is given by equations (1)-(6). Under special assumptions on parameter values with $c_{g}=-c_{d}=c$ and $\tau_{g}=\tau_{d}=\tau$, it can be simplified by summing equations (3) and (6), which yields

$$
\Delta P=2 P_{N}+C e^{-r t},
$$

where $C$ is solely determined by a perturbation and $r \equiv c / \tau$ quantifies the reactability of the system on perturbations and reflects how fast the power goes back to the normal state, i.e., the synchronization rate. For generation, $r$ indicates the comprehensive impacts of the marginal cost and response time constant on the generator's synchronization stability: the larger $c$ is, the more sensitive the generator becomes for power fluctuations or perturbations; the smaller $\tau$ is, the faster the generator responds for generation adjustment. With the increase of $r$, effects of the electricity price on the node dynamics become more and more pronounced. $r$ is independent of the stationary state, but its variance influences the changes in the size of the attracting basin of synchronization (see Supplementary Figure S2). The formation (9) is of course a strong simplification, yet acting as a cornerstone for the understanding of the intricate dynamics of interactions between price regulation and the physical network.

This reduced system (8) in the stationary state which has one stable fixed point, one saddle, and one stable limit cycle $[13,31]$. Perturbations affecting the system's dynamics can be induced by changes in generation and/or consumption, or by initial conditions of phase and frequency [32]. According to the definition of power system stability [5], the stability issue of power networks is related to physical disturbances, as load/generation variation, generator tripping, transmission line tripping, short circuit, etc. As a rule of thumb, power network dynamics emerge once it subjects to such perturbations, whereby the fluctuations of all the nodes' angular phase and speed occur. Thus, imposing physical perturbations instead of directly changing initial conditions of phase or frequency seems more natural and reasonable. Although [19] attempts to imitate short-circuit disturbances, it is still considerably simplified by ignoring the changes of initial conditions of most dimensions. Thus, in the sequel, all the perturbations take the form of setting step changes to power consumption or generation, affecting all-dimension dynamics simultaneously.

For small disturbances, the coupling term can be linearized in the vicinity of the equilibrium point [32]. Its maximal Lyapunov exponent is expected to be negative to stabilize the dynamics of price regulations, node responses, and networked dynamics. In this case, the equilibrium point is locally stable and hence the system will return back to the normal state after a certain perturbation. For nonsmall disturbances, depending on whether located in the attracting basin of the equilibrium point, the system will run normally or will go out of power. In what follows, we focus on large perturbations and quantify the power grid stability with the interpretation as the likelihood of the system's returning back to the normal state with respect to large perturbations (see Section 4).

Initially, system (8) is in the equilibrium state. As shown in Figures 1(b) and 1(c), at time $t=t_{0}$, it is then suddenly subjected to a physical disturbance, and the generation $P_{g}$ (in red) is perturbed far away from the normal state. After the sudden perturbation, the price $p$ regulates according to the linear correlation (1), and the consumption $P_{d}$ is forced to adjust its difference to generation, which can be taken as the second disturbance to the networked dynamics (7). The frequency difference $\Delta \omega$ is successively driven away from 0 . Depending on whether the perturbations are located inside or outside of the attracting basin of the synchronous state, the system will either return back (in Figure 1(b)) or will start wandering periodically to the wanted synchronous state (in Figure 1(c)). 


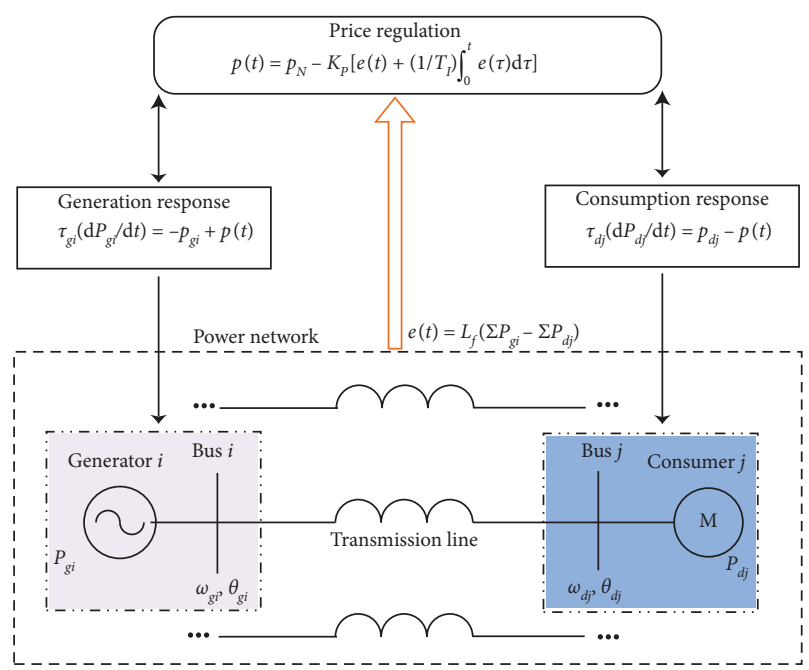

(a)

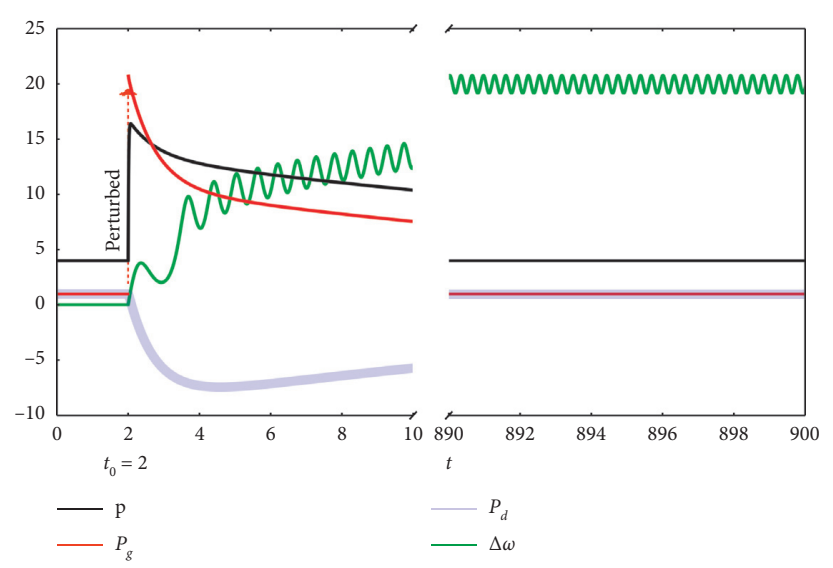

(c)

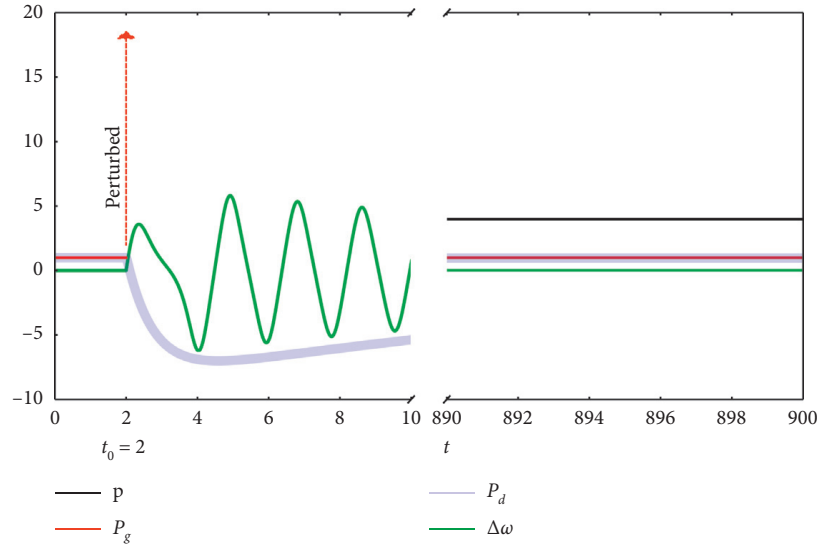

(b)

FIGURE 1: Interpretation of governing dynamics. (a) Schematic diagram for the interactions between different kinds of dynamics. In the twonode model (8), trajectories of the price $p$, generation $P_{g}$, and consumption $P_{d}$ are shown in (b) (resp. in (c)) given a perturbation suffered at time $t_{0}$ located inside (resp. outside) the attracting basin of the synchronous state. Parameters are given as $K_{P}=50, T_{l}=20, T_{D}=0.5$, $b_{g}=3, c_{g}=1, b_{d}=5, c_{d}=-1, f_{N}=1, M=2.0, \alpha=0.1, K=8, \tau_{g}=0.5$, and $\tau_{d}=1.0$.

To quantify the effects of electricity markets on power system stability, without varying the location of equilibrium points, we calculate the system's antidisturbance capability (ADC) with respect to the synchronization rate $r$. As the stationary state of systems (1)-(6) is $\tau$-independent but $c$-dependent, $r$ is varied via the reaction time $\tau$. ADC vs. $r$ is shown in Figure 2(a). The projections of two trajectories consisting of one returning back to the normal state and the other oscillating periodically in the phase space $(\Delta \theta, \Delta \omega)$ are shown in the inset. Simulations are conducted with $T=$ 400 perturbations for $P_{g}$ randomly selected within the range of $[-20,20]$ at each $r$. System stability is approximately estimated according to equation (11) with the standard error $e<0.03$. Intuitively, strengthening electricity markets might enhance system's stability.

While given $C / r$ constant, various transitions could occur depending crucially on the value of $r$. For high values of $r$, the system exhibits robustness against perturbations and has high volumes $(\mathrm{ADC})$ of basins of attraction. If starting from large values of $r$, ADC increases smoothly and then gradually becomes globally stable with ADC $=1$ for further increases of $r$. For small values of $r$, synchronization rate decreases and this induces the shrinking of the attraction basin, as shown in Figure 2(b). Interestingly, if starting from a rather small value of $r$, ADC first decreases and approaches a minimum value, and only then it increases smoothly (for detailed interpretation, see SN 2). This can also be reflected by the emerging red region in the low-left corner of Figure 2(b).

This also indicates that enhancing market effects may undermine power grid stability under certain circumstances, which needs to be avoided for both power network planning and operation. These results are obtained from the lowdimensional system, the simple two-node model of a strong simplification. However, can similar results be observed in high-dimensional systems?

2.3. China Southern Power Grid (CSG). Hence, we now turn to the CSG (see Section 4 for details), which consists mainly of thermal, hydro, and nuclear generators. The specific 


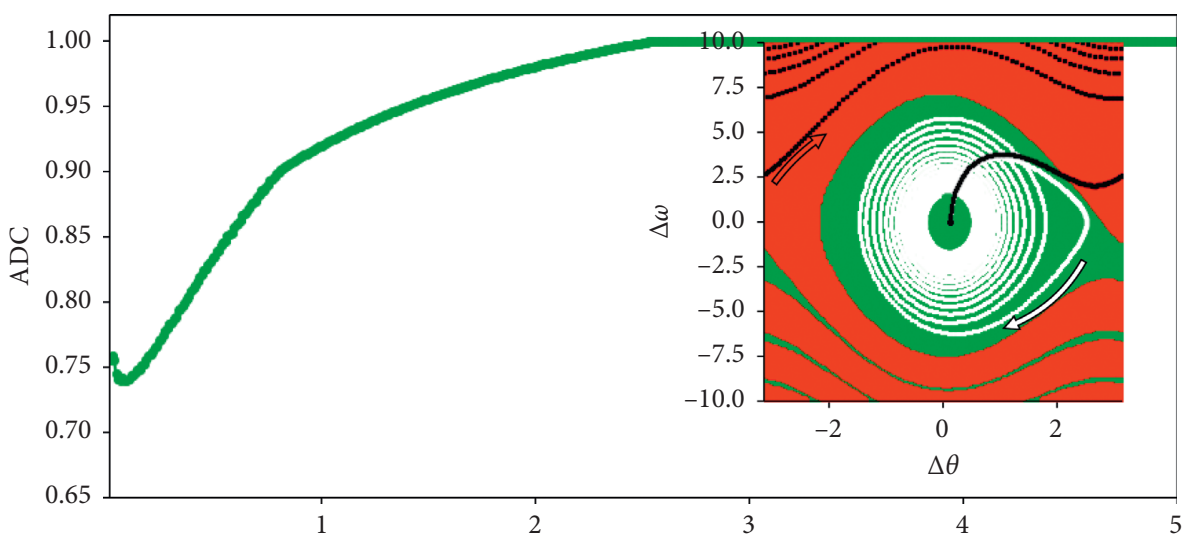

(a)

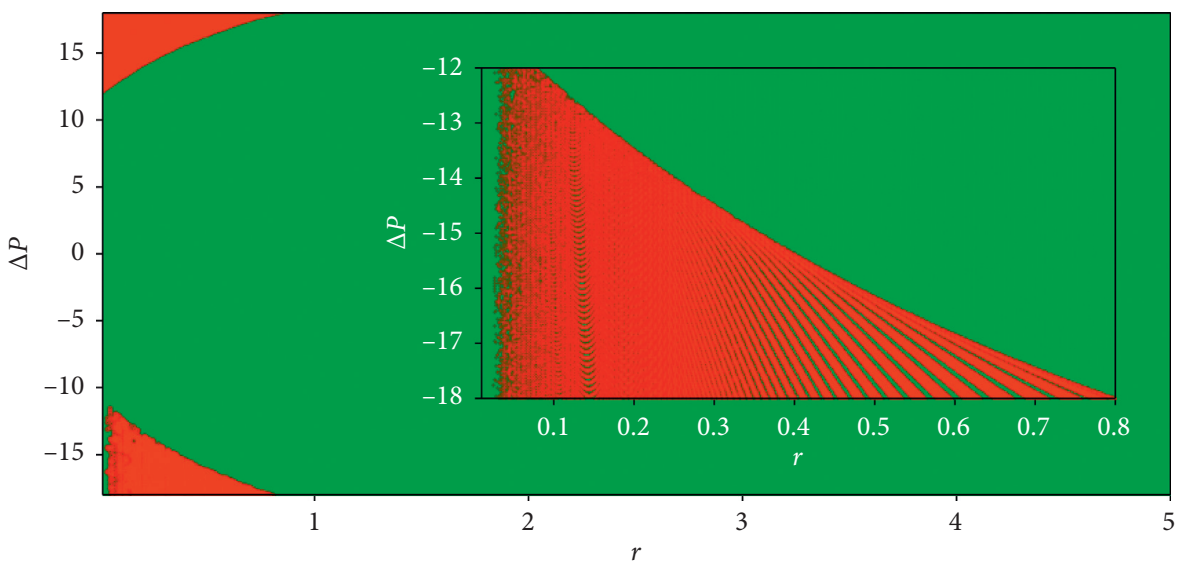

(b)

FIgURE 2: Stability of the two-node model. (a) The system antidisturbance capability ADC with respect to the synchronization rate $r$. Parameters are the same as in Figure 1 but with varied $\tau_{g}$. In the inset, two kinds of trajectories are projected in the phase space $(\Delta \theta, \Delta \omega)$, consisting of one leading the two-node model (8) back to the synchronous state colored in white and the other driving the system wandering periodically colored in black. (b) Perturbations located inside of the attracting basin are colored in green and outside of the region are colored in red as a function of $r$. Zoom-in part illustrate the complex structure of the local attracting region.

response characteristics of different types of power generators are taken into main consideration. Additionally, these three kinds of plants, especially thermal and hydro ones, have distinct environmental impacts. Particularly, thermal plants in southern China are mainly based on coal combustion, which constitutes the main source of contamination and carbon emission in CSG, while hydro ones generally produce power in a clean manner. Based on the behaviors and amounts of consumption, consumers are classified into 3 typical categories: industrial, commercial, and residential loads. All nodes' categories and topological connections are listed in Supplementary Tables S1 and S2. Both generators and consumers evolve, respectively, on equations (3) and (6) yet with different characteristics (see Tables 1 and 2), except for nuclear plants which are independent of price regulations. As stated in Section 4, each node stands for an aggregated plant or consumer, corresponding to a series of actual generators or loads. Under this circumstance, perturbations of $P_{g}$ on individual loads represent sudden tripping of small-scale generators in practice or the total fluctuation of power generation of the corresponding aggregated plant. In what follows, we especially address these
TABLE 1: Parameters of generation response.

\begin{tabular}{lccc}
\hline Categories of plants & $\tau_{g i} / s$ & $b_{g i}$ & $c_{g i}$ \\
\hline Thermal & $3 \sim 30$ & $1.3 \sim 4.8$ & $0.3 \sim 3.6$ \\
Hydro & $0.2 \sim 10$ & $1.2 \sim 3.4$ & $0.2 \sim 1.2$ \\
Nuclear & $\infty$ & - & - \\
\hline
\end{tabular}

TABLE 2: Parameters of consumption response.

\begin{tabular}{lccc}
\hline Categories of loads & $\tau_{d j} / s$ & $b_{d j}$ & $c_{d j}$ \\
\hline Industrial & $8 \sim 60$ & $2.2 \sim 8$ & $-1 \sim-0.2$ \\
Commercial & $5 \sim 20$ & $3.4 \sim 8.6$ & $-1.5 \sim-0.6$ \\
Residential & $0.2 \sim 10$ & $4.2 \sim 10$ & $-3 \sim-1.2$ \\
\hline
\end{tabular}

two aspects: effects of electricity markets on the grid stability and on environmental pollution.

(i) Let us now first revisit generation/consumption dynamics and settings of physical disturbances referred above, so as to quantify the effects of electricity markets on the grid stability ADC as a function of 
the synchronization rate $r$. Synchrony in the grid is achieved when the maximal absolute deviation of the frequencies $\omega_{i}$ vanishes in time. The networked model (7) exhibits the essential feature of the system, the coexisting stationary state, as soon as it comprises of the two-node model [12]. After perturbation, individual nodes behave differently. And after some transition, actually only nodes around the birthplace of the perturbation are observably driven away from the synchronous state, while others remain close to it, as depicted in Figure 3(a), where the perturbation is imposed on node 0 .

Following the process outlined in the previous lowdimensional model, concretely, simulations are conducted via perturbing power generation $\left(P_{g}\right)$ of thermal and hydro plants within the range of $[-20,20]$. Our results suggest that, as depicted in Figure 3(b), variation of ADC with respect to $r$ illustrates qualitatively a similar trend (as in Figure 2(a)): sinking first and then rising subsequently. For each node $i$, we estimate single-node stability $\mathrm{ADC}_{i}$ which expresses the probability of all nodes returning back to the normal state after the $i$ th node has been subjected to small and even large perturbations. Projection of $\mathrm{ADC}_{i}$ on each node $i$ with its color strength proportional to $1-\mathrm{ADC}_{i}$ is shown in Figure 4. Clearly, the low degree nodes (in dark red) are likely to undermine power grid stability $[33,34]$.

(ii) Next we seek to decarbonize the power plants. Would it be possible to decrease the thermal generation and to increase the hydrogeneration, while simultaneously keeping the power grid still stable and robust? On the assumption that the stationary state of price $p$ remains as a constant, in order to achieve this goal, one might improve the economic efficiency of hydro and thermal generators, e.g., decreasing its marginal-cost coefficient $c_{g}$ by an equipment upgrade and technological improvement.

Assume that both hydro and thermal generation units in CSG are updated with more advanced technology, corresponding to the parameter shift of $c_{g}$, which are artificially decreased by $30 \%$ and $10 \%$, respectively. This induces the reallocation of power flows. As shown in the insets of Figure 4, our results suggest that after improving the economic efficiency, the thermal generation over the total generation is decreased from $55 \%$ to $47 \%$, whereas the hydrogeneration's percentage is increased from $39 \%$ to $48 \%$. The price remains almost constant as expected. This of course further affects the system's performance, especially its stability against large perturbations. Therefore, we re-estimate the single-node stability $\mathrm{ADC}_{i}^{\prime}$ for all nodes listed in Supplementary Tables S1 and S2. We find that few nodes' stability is even enhanced (see green-colored values in Supplementary Table S1), while some nodes' stability is decreased (in red). But the whole power grid still remains fairly stable.
Therefore, on the whole, both tasks of decarbonization of power production and maintenance of system capability are accomplished in this way. However, the results also reveal that the single-node stability of some individual nodes is somewhat decreased. It is crucial to identify overloaded nodes to avoid cascading failures $[35,36]$. In practice, the update process of equipment on these nodes is suggested to be avoided or deferred, so that a better trade-off between the decarbonization and system stabilization can be achieved.

\section{Discussion}

We have investigated effects of real-time price-based frequency regulation on power grid stability against even large perturbations. Specifically, in the coexistence regime of the two-node model and in a linear-stable regime of the markets (see SN 2), we have found that the system stability can be undermined via increasing the control of the electricity price mechanism. The results remain valid in the rather large China Southern Power Grid with more practical scenarios, composing of three types of generators (thermal, hydro, and nuclear plants) and of consumers (residential, commercial, and industrial loads). Of course, power grid stability analysis is rather complicated and can be influenced by various aspects, e.g., parameter settings, ranges of perturbations, and network topologies. Therefore, we have validated the results in the two-node model with an even larger range of perturbations (see SN 2), in the northern European power grid (see SN 3) and additionally, in the China Southern Power Grid with a different power allocation with a single type of generator and of consumer (see SN 4). All these simulations exhibit the local minimum phenomenon of system antidisturbance capability with a continuous increase of the synchronization rate.

Generators react accordingly with different characteristics and have different environmental impacts. Given the specific scenario in the China Southern Power Grid, we have demonstrated a simple but feasible approach, involving updates of hydro and thermal generation units, to achieve the dual target of decarbonization and stability maintenance. Our experiments confirm the possibility of reducing the thermal production's percentage and increasing the percentage of hydroproduction, and meanwhile the grid remains fairly stable afterwards.

What remains to be investigated next? Primarily, renewable energy generation, including wind power and solar energy, and more realistic models of consumers instead of synchronous motors deserve further research efforts [37]. Additionally, several efforts should be taken into account, e.g., more detailed network structures and the relationship between the overall decarbonization benefits and the system's basin of attraction.

\section{Methods and Materials}

4.1. Stability Measure. We take the following general approach to quantify system antidisturbance capability (ADC), which is intended to evaluate how stable the synchronous 


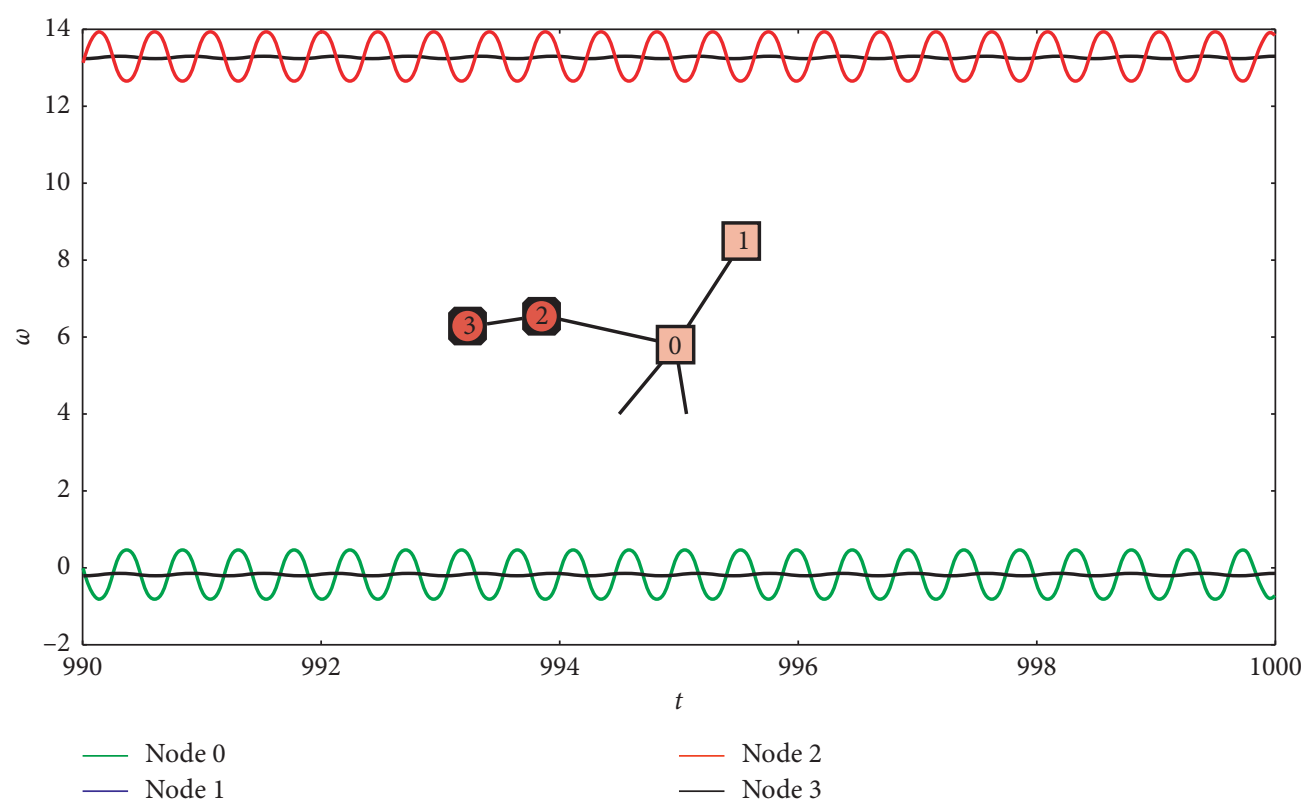

(a)

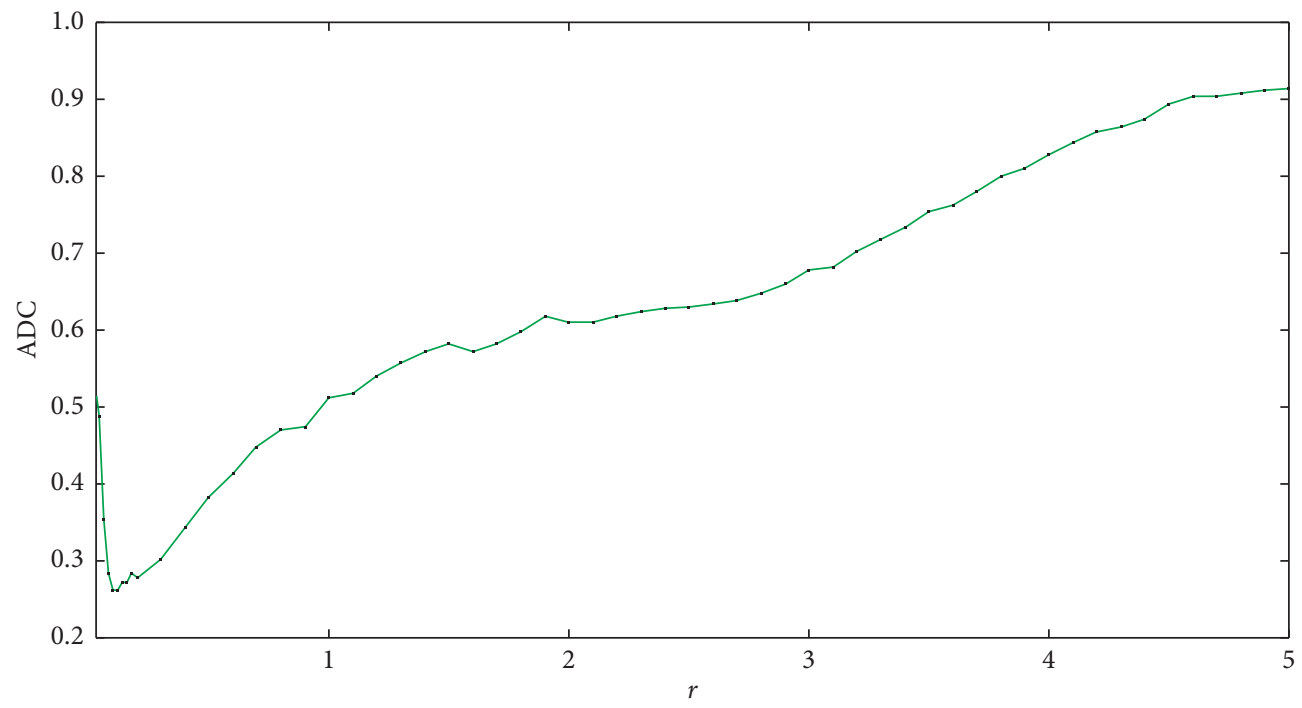

(b)

FIgURE 3: Stability of the CSG. (a) Time series of the snippet from the China Southern Power Grid with a one-time artificial perturbation located at node 0 . In the stationary state, nodes 2 and 3 are driven periodically away from the synchronous state, while nodes 0 and 1 remain close to the state. (b) System antidisturbance capability ADC with respect to the synchronization rate $r$. Parameters are as follows: $K_{P}=50$, $T_{l}=20, T_{D}=0.5, b_{g}=3, c_{g}=1, b_{d}=5, c_{d}=-1, f_{N}=1, M=2.0, \alpha=0.1, K=8, \tau_{g}=0.5$, and $\tau_{d}=1.0$. For residential loads $\tau_{d}=0.2$, commercial loads $\tau_{d}=5.0$, and industrial loads $\tau_{d}=8.0$, with varying the reaction rate of generators and therefore $r=1 / \tau$.

state is against large physical perturbations for generations and/or consumption $P$, especially defined as

$$
\mathrm{ADC}=\int \chi(P) \rho(P) \mathrm{d} P,
$$

where $\chi(P)$ is an indicator function with $\chi(P)=1$, if $P$ belongs to the attracting basin of the equilibrium point and $\chi(P)=0$, otherwise; $\rho$ is a density function normalized as $\int \rho(P) \mathrm{d} P=1$. Its magnitude suggests how likely the system comes back to the stable fixed point after suffering from large perturbations. Numerical Monte Carlo methods are employed to estimate the likelihood. In particular, initially the system is synchronized and we draw $T$ random initial conditions according to the density function $\rho(P)$, solve the system by numerical integration, and then count the number of initial conditions driving the system back to the synchronous state. This yields

$$
\mathrm{ADC} \approx \frac{S}{T}
$$

with standard error $e=\sqrt{(\mathrm{ADC}(1-\mathrm{ADC})) / T}$. Note that perturbations are imposed physically on power production or demand of individual nodes instead of the changing phase and frequency as before in [19]. 


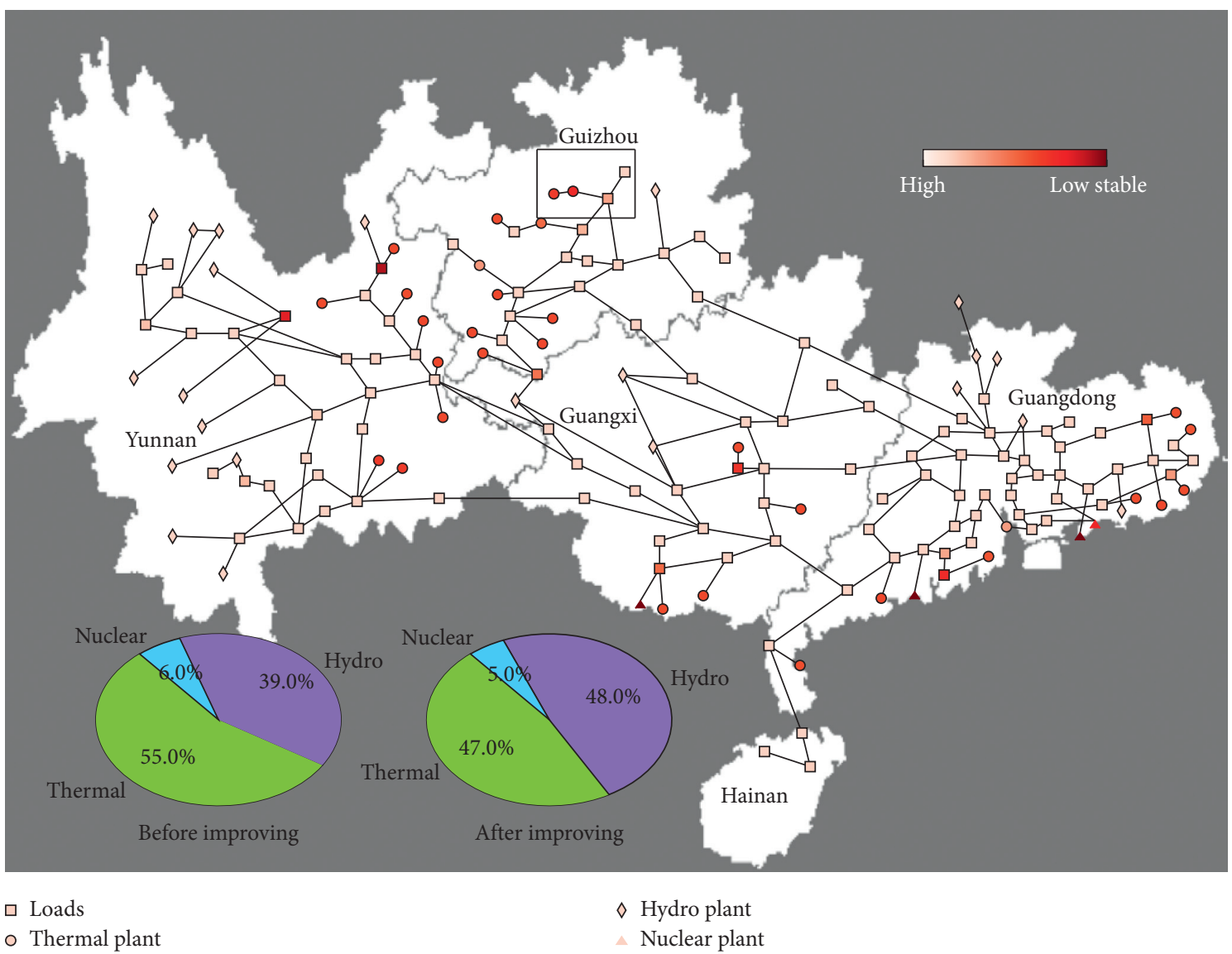

Figure 4: Stability and decarbonization of the CSG. Single-node stability on the China Southern Power Grid (nodes' type is shown in supplementary table S1, and edges are listed in supplementary table S2). The two pie charts show the percentage of the three kinds of generator plants before and after improving the economic efficiency of hydropower plants by decreasing their $c_{g}$ from 1 to 0.7 and decreasing thermal $c_{g}$ from 1 to 0.9 . Parameters are the same as in Figure 3 with $\tau_{g}=3.0$ for thermal plants and $\tau_{g}=0.2$ for hydroplants.

4.2. Topology of China Southern Power Grid. China Southern Power Grid (CSG) is one of the two large-scale power grids in China, consisting of Yunnan, Guizhou, Guangxi, Guangdong, and Hainan provincial subnetworks. As shown in Figure 4, the main topology of the bulk alternating-current (AC) electric network of CSG is extracted for this case study. This real-world network preserves the primary structure and characteristics of the whole system. On the basis of the planning data of the Dispatching Center of CSG in 2014, it is simplified by the following equivalences. Similar generators in one location are equivalently represented by a large generator [38]; to avoid trivial and complicated network modeling, all the low-voltage distribution networks are aggregated into corresponding high-voltage substations. The final reduced network includes 57 generators and 111 loads, connected by 194 edges. In particular, the generators are mainly comprised of hydro, thermal, and nuclear plants, whose deployments are based on actual situations. It is because these three forms of generations still occupy a major proportion of the total power generation in southern China, though an increasing penetration of wind power and photovoltaic generation has been witnessed in the last decade. The loads also involve industrial, commercial, and residential categories, and, from the perspective of high-voltage grids, each load node contains all the three.

4.3. Parameters Setting. Within the three categories of generation in CSG, thermal and hydro generators are likely to respond to the real-time price, while the outputs of nuclear ones are not affected by price at any time, due to safety consideration and technical restriction. In terms of thermal plants, owing to the inertia of thermodynamic systems and technical requirements of mild output, their ramp rates are relatively limited; thus, their $\tau_{g i}$ is fairly large. On the contrary, hydroplants are permitted to operate in a more flexible way, which means that a smaller $\tau_{g i}$ is approachable. Taking into account the economic cost and environmental expense in China [39], the monomial coefficient $c_{g i}$ of thermal generation is supposed to be about $1.5 \sim 3$ times larger than that of the hydrocounterpart. Given specific $c_{g i}, b_{g i}$ is determined by the rated price $p_{N}$ and output $P_{g i N}$ at the stationary status. Concrete ranges of $\tau_{g i}$, $b_{g i}$, and $c_{g i}$ are summarized in Table $1[24,39,40]$.

In the light of practical experiences, concrete ranges of parameters of the 3 typical kinds of consumers are listed in Table $2[24,39,40]$. For a large factory, safety and continuity 
of the production process should be guaranteed first, and so as to ensure the quality of its products, it is not that willing to change its amount of power consumption even given a certain degree of marginal benefit. However, residents care more about the varying price, and they are most likely to adjust their consumption once receiving the latest price information, aiming at gaining larger benefits. As for shopping malls or supermarkets, sometimes (during the slack period) they tend to react to the varying price timely, while sometimes (during the peak period) not. Therefore, from a comprehensive point of view, residential consumers are deemed to be most sensitive to price variation, commercial ones are relatively mediate, whereas industrial users are least sensitive.

\section{Data Availability}

The China Southern Power Grid and other data used to support the findings of this study are available from the corresponding author upon request.

\section{Conflicts of Interest}

The authors declare no conflicts of interest.

\section{Authors' Contributions}

P.J. and L.Z. designed the study and prepared the manuscript. L.Z. prepared the data. W.L. and P.J. carried out the analysis. All authors discussed the results and contributed to editing the manuscript. J.K., W.L., and C.L. supervised the study.

\section{Acknowledgments}

The authors acknowledge the National Key R\&D Program of China (Grant no. 2018YFB0904500), Natural Science Foundation of Shanghai, the Program for Professor of Special Appointment (Eastern Scholar) at Shanghai Institutions of Higher Learning, and NSFC 269 (Grant no. 11701096), National Science Foundation of China under Grant no. 61773125. L.Z.'s work was supported by the Research Grants Council of Hong Kong under the Themebased Research Scheme (TRS) through Project no. T23-701/ 14-N. C.L.'s work was supported by National Natural Science Foundation of China under Grant nos. 51677097 and U1766214. W.L. was supported by the Research Institute of Intelligent and Complex Systems, Fudan University, China, the National Science Foundation of China (NSFC) (Grant nos. 11925103 and 61773125), the National Key R\&D Program of China (Grant no. 2018YFC0116600), and the Science and Technology Commission of Shanghai Municipality (Grant no. 18DZ1201000). J. K was supported by the project RF Goverment Grant 075-15-2019-1885.

\section{Supplementary Materials}

I Supplementary note 1: derivation of marginal cost. II Supplementary note 2: two-node model. III. Supplementary note 3: China southern grid. IV Supplementary note 4: northern European power grid. (Supplementary Materials)

\section{References}

[1] O. Edenhofer and R. Pichs-Madruga, IPCC Special Report on Renewable Energy Sources and Climate Change Mitigation, Cambridge University Press, Cambridge, UK, 2011.

[2] D. Butler, "Energy efficiency: super savers: meters to manage the future," Nature, vol. 445, no. 7128, pp. 586-588, 2007.

[3] M. Amin, "Energy: the smart-grid solution," Nature, vol. 499, no. 7457, pp. 145-147, 2013.

[4] B. Schäfer, M. Matthiae, M. Timme, and D. Witthaut, "Decentral smart grid control," New Journal of Physics, vol. 17, no. 1, Article ID 015002, 2015.

[5] P. Kundur, J. Paserba, V. Ajjarapu et al., "Definition and classification of power system stability IEEE/CIGRE joint task force on stability terms and definitions," Power Systems, IEEE Transactions, vol. 19, no. 3, pp. 1387-1401, 2004.

[6] A. Arenas, A. Díaz-Guilera, J. Kurths, Y. Moreno, and C. Zhou, "Synchronization in complex networks," Physics Reports, vol. 469, no. 3, pp. 93-153, 2008.

[7] F. A. Rodrigues, T. K. D. Peron, P. Ji, and J. Kurths, "The kuramoto model in complex networks," Physics Reports, vol. 610, pp. 1-98, 2016.

[8] J. A. Acebrón, L. L. Bonilla, C. J. Pérez Vicente, F. Ritort, and R. Spigler, "The kuramoto model: a simple paradigm for synchronization phenomena," Reviews of Modern Physics, vol. 77, no. 1, pp. 137-185, 2005.

[9] T. Nishikawa and A. E. Motter, "Comparative analysis of existing models for power-grid synchronization," New Journal of Physics, vol. 17, no. 1, Article ID 015012, 2015.

[10] F. Dörfler and F. Bullo, "Synchronization in complex networks of phase oscillators: a survey," Automatica, vol. 50, no. 6, pp. 1539-1564, 2014.

[11] D. J. Hill and G. Chen, "Power systems as dynamic networks," in Proceedings of the 2006 IEEE International Symposium on Circuits and Systems, IEEE, Island of Kos, Greece, May 2006.

[12] M. Rohden, A. Sorge, M. Timme, and D. Witthaut, "Self-organized synchronization in decentralized power grids," Physical Review Letters, vol. 109, no. 6, Article ID 064101, 2012.

[13] P. Ji, T. K. D. Peron, P. J. Menck, F. A. Rodrigues, and J. Kurths, "Cluster explosive synchronization in complex networks," Physical Review Letters, vol. 110, no. 21, Article ID 218701, 2013.

[14] S. Olmi, A. Navas, S. Boccaletti, and A. Torcini, "Hysteretic transitions in the kuramoto model with inertia," Physical Review E, vol. 90, no. 4, Article ID 042905, 2014.

[15] P. Ji, W. Lu, and J. Kurths, "Stochastic basin stability in complex networks," EPL (Europhysics Letters), vol. 122, no. 4, p. 40003, 2018.

[16] F. Dai, S. Zhou, T. Peron, W. Lin, and P. Ji, "Interplay among inertia, time delay, and frustration on synchronization dynamics," Physical Review E, vol. 98, no. 5, Article ID 052218, 2018.

[17] L. Chen, P. Ji, D. Waxman, W. Lin, and J. Kurths, "Effects of dynamical and structural modifications on synchronization," Chaos: An Interdisciplinary Journal of Nonlinear Science, vol. 29, no. 8, Article ID 083131, 2019.

[18] P. J. Menck, J. Heitzig, N. Marwan, and J. Kurths, "How basin stability complements the linear-stability paradigm," Nature Physics, vol. 9, no. 2, pp. 89-92, 2013.

[19] P. J. Menck, J. Heitzig, J. Kurths, and H. J. Schellnhuber, "How dead ends undermine power grid stability," Nature Communications, vol. 5, 2014. 
[20] B. K. Poolla, S. Bolognani, and F. Dorfler, "Optimal placement of virtual inertia in power grids," IEEE Transactions on Automatic Control, vol. 62, no. 12, pp. 6209-6220, 2017.

[21] P. Kundu, P. Khanra, C. Hens, and P. Pal, "Transition to synchrony in degree-frequency correlated sakaguchi-kuramoto model," Physical Review E, vol. 96, no. 5, Article ID 052216, 2017.

[22] P. Kundu, C. Hens, B. Barzel, and P. Pal, "Perfect synchronization in networks of phase-frustrated oscillators," $E P L$ (Europhysics Letters), vol. 120, no. 4, p. 40002, 2018.

[23] B. Schäfer, C. Beck, K. Aihara, D. Witthaut, and M. Timme, "Non-gaussian power grid frequency fluctuations characterized by lévy-stable laws and superstatistics," Nature Energy, vol. 3, no. 2, pp. 119-126, 2018.

[24] A. W. Berger and F. C. Schweppe, "Real time pricing to assist in load frequency control," IEEE Transactions on Power Systems, vol. 4, no. 3, pp. 920-926, 1989.

[25] Y. G. Jin, S. Y. Lee, S. W. Kim, and Y. T. Yoon, "Designing rule for price-based operation with reliability enhancement by reducing the frequency deviation," IEEE Transactions on Power Systems, vol. 28, no. 4, pp. 4365-4372, 2013.

[26] F. Alvarado, "The stability of power system markets," IEEE Transactions on Power Systems, vol. 14, no. 2, pp. 505-511, 1999.

[27] A. R. Bergen and V. Vittal, Power System Analysis, PrenticeHall, Upper Saddle River, NJ, USA, 2nd edition. 2002.

[28] R. Baldick, "Electricity market equilibrium models: the effect of parametrization," IEEE Transactions on Power Systems, vol. 17, no. 4, pp. 1170-1176, 2002.

[29] G. Filatrella, A. H. Nielsen, and N. F. Pedersen, "Analysis of a power grid using a kuramoto-like model," The European Physical Journal B, vol. 61, no. 4, pp. 485-491, 2008.

[30] F. Dörfler, M. Chertkov, and F. Bullo, "Synchronization in complex oscillator networks and smart grids," Proceedings of the National Academy of Sciences, vol. 110, no. 6, pp. 20052010, 2013.

[31] S. H. Strogatz, Nonlinear Dynamics and Chaos: With Applications to Physics, Biology, Chemistry, and Engineering, Westview Press, Boulder, CO, USA, 2014.

[32] J. Machowski, J. Bialek, and J. Bumby, Power System Dynamics: Stability and Control, John Wiley \& Sons, Hoboken, NJ, USA, 2011.

[33] A. E. Motter and Y.-C. Lai, "Cascade-based attacks on complex networks," Physical Review E, vol. 66, no. 6, Article ID 065102, 2002.

[34] S.-P. Zhang, Z.-G. Huang, J.-Q. Dong, D. Eisenberg, T. P. Seager, and Y.-C. Lai, "Optimization and resilience of complex supply-demand networks," New Journal of Physics, vol. 17, no. 6, Article ID 063029, 2015.

[35] R. Cohen and S. Havlin, Complex Networks: Structure, Robustness and Function, Cambridge University Press, Cambridge, UK, 2010.

[36] J. Zhao, D. Li, H. Sanhedrai, R. Cohen, and S. Havlin, "Spatiotemporal propagation of cascading overload failures in spatially embedded networks," Nature Communications, vol. 7, 2016.

[37] P. Milan, M. Wächter, and J. Peinke, "Turbulent character of wind energy," Physical Review Letters, vol. 110, no. 13, p. 138701, 2013.

[38] J. Zhang, C. Y. Chung, C. Lu, K. Men, and L. Tu, “A novel adaptive wide area PSS based on output-only modal analysis," IEEE Transactions on Power Systems, vol. 30, no. 5, pp. 2633-2642, 2015.
[39] "World Energy Perspective, https://www.worldenergy.org/wpcontent/uploads/2013/09/WEC_J1143_CostofTECHNOLOGIES _021013_WEB_Final.pdf.

[40] A. Jokic, E. H. M. Wittebol, and P. P. J. Van den Bosch, "Dynamic market behavior of autonomous network-based power systems," European Transactions on Electrical Power, vol. 16, no. 5, pp. 533-544, 2006. 